\title{
Radiative transfer in cylindrical threads with incident radiation
}

\section{2D azimuth-dependent case}

\author{
P. Gouttebroze
}

\author{
Institut d'Astrophysique Spatiale, Univ. Paris XI/CNRS, Bât. 121, 91405 Orsay Cedex, France \\ e-mail: goutte@ias.u-psud.fr
}

Received 3 November 2004 / Accepted 5 January 2005

\begin{abstract}
A method is proposed for the solution of NLTE radiative transfer equations in long cylinders with an external incident radiation that varies with direction. This method is designed principally for the modelling of elongated structures imbedded in the solar corona (loops, prominence threads). The radiative transfer problem under consideration is a $2 \mathrm{D}$ one, since the source functions and absorption coefficients vary with both distance to axis and azimuth. The method is based on the general principles of finite-differences and accelerated $\Lambda$-iteration. A Fourier series is used for interpolation in azimuth. The method is applied to a line emitted by a two-level atom with complete frequency redistribution. Convergence properties of the method and influence of the inclination angle on the source function are discussed.
\end{abstract}

Key words. methods: numerical - radiative transfer - line: profiles - line: formation - Sun: corona

\section{Introduction}

This is the third paper dedicated to solving NLTE radiative transfer equations in elongated cylindrical structures with external incident radiation. The principal aim of this project is the modelling of the thread-like objects produced by magnetic fields and visible in the solar atmosphere. At the beginning (Gouttebroze 1990, hereafter G90), we proposed a semianalytical method to treat the two-level atom problem in the Eddington approximation (by "semi-analytical", we mean that there was no discretization in radius, but only in frequency). In this method, source functions and intensities were expressed as Bessel function expansions; however, the method was of little practical use, since it suffered from many restrictions (homogeneous medium, two-level atom, etc.).

Later on (Gouttebroze 2004, hereafter Paper I), we proposed a method to treat the general one-dimension (1D) cylindrical problem, allowing multilevel atoms, variations of medium properties along the radius, and departures from Eddington approximation. It was applied to a hydrogen-filled medium with a self-consistent determination of electron density. This method may be used to perform a realistic modelling of approximately vertical threads seen above the solar limb, taking into account thread-corona transition regions.

In the present paper, we generalize the previous method to two dimensions (2D), allowing variations of the radiation field and medium properties both in radius and azimuth. However, we do not treat here the case of a fully arbitrary incident radiation, but assume that this radiation is emitted by a sphere representing the solar surface. This implies a symmetry, which allows the reduction of the azimuth grid by a factor of 2 . The method is formulated in Sect. 2, and numerical results for a two-level atom are discussed in Sect. 3. The extension to multilevel atoms, necessary for a realistic modelling of threads of any direction imbedded in the solar corona, will be treated in subsequent papers.

\section{Formulation}

\subsection{Transfer equation}

We use the same notations as in Paper I; i.e. the running point is represented by three cylindrical coordinates: $r$ (distance to the axis), $\psi$ (azimuth), and $z$ (distance along the axis). The direction of the ray is given by two angles $\theta$ and $\zeta: \theta$ (from $0^{\circ}$ to $180^{\circ}$ ) is the angle between a parallel to the axis and the ray, while $\zeta$ (from $-90^{\circ}$ to $90^{\circ}$ ) is an azimuthal angle (cf. Fig. 1). In this paper, we consider a $2 \mathrm{D}$ problem $(r, \psi)$, with invariance by translation along the axis. Taking the three-dimension radiative transfer equation and dropping the $z$-derivative, we obtain

$\sin \theta\left[\cos \zeta \frac{\partial I_{v}}{\partial r}+\frac{\sin \zeta}{r}\left(\frac{\partial I_{v}}{\partial \psi}-\frac{\partial I_{v}}{\partial \zeta}\right)\right]=-\kappa_{v}\left(I_{v}-S_{v}\right)$

where, as usual, $I_{v}$ is the intensity, $\kappa_{v}$ the absorption coefficient, and $S_{v}$ the source function. Another important relation is the transfer equation along a ray crossing the cylinder

$Y_{v}-\frac{\sin ^{2} \theta}{\kappa_{v}} \frac{\partial}{\partial h}\left(\frac{1}{\kappa_{v}} \frac{\partial Y_{v}}{\partial h}\right)=S_{v}$ 


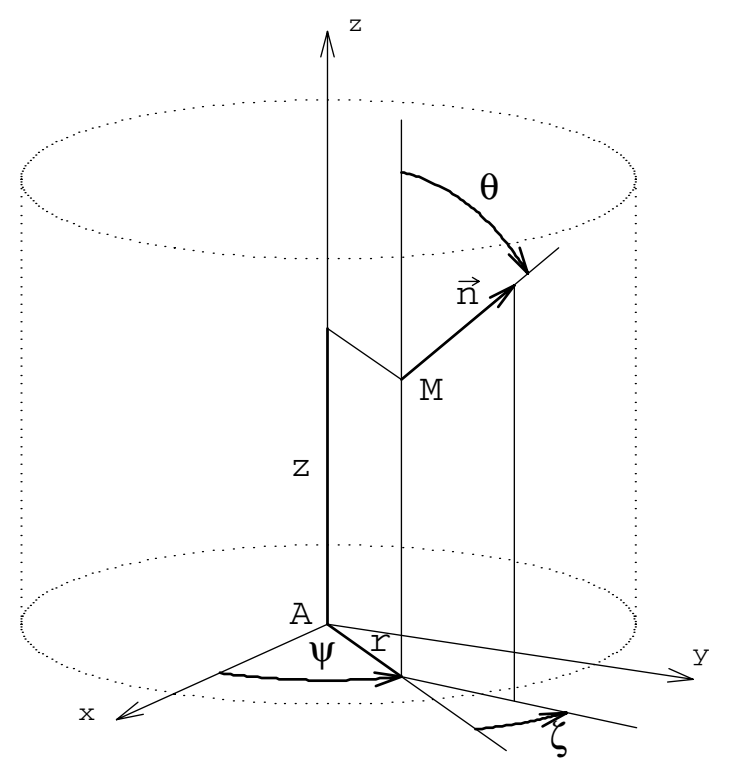

Fig. 1. System of coordidates for the cylinder: the location of the running point is defined by $r, \psi$ and $z$, the direction of the ray by $\theta$ and $\zeta$.

where $Y_{v}$ is the average of intensities in both directions and $h$ the projection, on the $(\theta=0)$ plane, of the abscissa of the running point along the ray.

For the computation of intensities along the ray, Eq. (2) must be complemented by boundary conditions. If $I_{1}^{\text {inc }}$ and $I_{2}^{\text {inc }}$ are the incident intensities at each end of the ray, and if the entrance and exit points correspond to abcissae $h_{1}$ and $h_{\max }$, respectively, the boundary conditions may be written

$\left.\begin{array}{l}{\left[Y_{v}-\frac{\sin \theta}{\kappa_{v}} \frac{\partial Y_{v}}{\partial h}\right]_{h=h_{1}}=I_{1}^{\mathrm{inc}}} \\ {\left[Y_{v}+\frac{\sin \theta}{\kappa_{v}} \frac{\partial Y_{v}}{\partial h}\right]_{h=h_{\max }}=I_{2}^{\mathrm{inc}}}\end{array}\right\}$.

\subsection{Incident radiation}

The cylinder is assumed to receive radiation from a sphere, which represents the Sun. At a given frequency, the intensity emitted by the sphere is a function $I_{\mathrm{em}}(\mu)$, where $\mu$ is the cosine of the angle between the local vertical and the emission direction. The radius of the cylinder is supposed to be small with respect to both solar radius and altitude, so that the incident intensity is a function of the azimuth $\psi$ and of the direction angles $\theta$ and $\zeta$, only. By convention, we assume that $\psi$ vanishes when the running point of the cylinder surface is turned towards the sphere, so that the incident radiation is maximum. In addition, the radiation field received by the cylinder depends on two geometrical parameters: $H$, the altitude above the solar surface, and $\alpha$, the angle of the axis of the cylinder with the vertical (see Fig. 2). For reasons of symmetry, it is sufficient to consider values of $\alpha$ between 0 and $\pi / 2$. Let $A$ be the running point of the cylinder surface (azimuth $\psi$ ) and $O$ the center of the sphere.

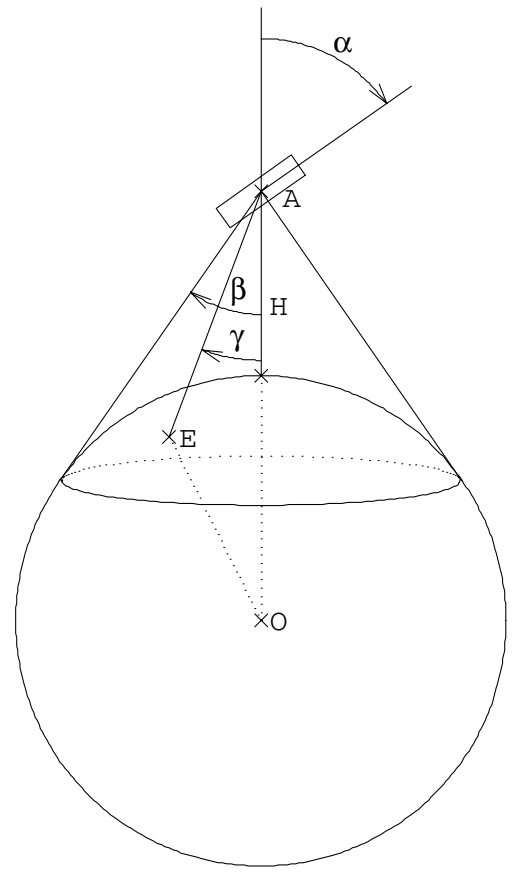

Fig. 2. System formed by the cylinder and the sphere: distance $H$ and inclination angle $\alpha$. The angles $\beta$ and $\gamma$ are used in computing incident intensities.

The cone of summit $A$ tangent to the sphere is characterized by the half-angle of aperture

$\beta=\arcsin \left(\frac{R_{\odot}}{R_{\odot}+H}\right)$

where $R_{\odot}$ is the radius of the sphere.

Now, we consider the ray from $A$ defined by direction angles $\theta$ and $\zeta$. An elementary calculation yields the angle $\gamma$ between this ray and the axis $A O$

$\gamma=\arccos [\sin \alpha \sin \theta \cos (\psi+\zeta)-\cos \alpha \cos \theta]$.

If $\gamma$ is larger than $\beta$, the ray does not cross the sphere, so that the incident intensity vanishes. For $\gamma<\beta$, the incident intensity is equal to that emitted at the surface of the sphere. Thus we have

$I^{\mathrm{inc}}=\left\{\begin{array}{cc}I_{\mathrm{em}}\left(\sqrt{1-\frac{\sin ^{2} \gamma}{\sin ^{2} \beta}}\right) & \text { if } \gamma<\beta \\ 0 & \text { if } \gamma>\beta .\end{array}\right.$

For radiative transfer computations, we use coarse grids in $\theta$ and $\zeta$, for instance 4 points for each of these variables in the present case. A direct use of the preceding formula with the coarse grids would produce some inaccuracy, as a result of discontinuities in the radiation field near the solar limb. To obtain more accurate values, the intensities are computed for a fine grid, e.g. $100 \times 100$ for the half-space, and subsequently averaged over the 16 solid angles surrounding each $(\theta, \zeta)$ point on the coarse grid. These intensities are represented in Fig. 3 for three different values of inclination angle $\alpha$. Equations (5) and (6) are given for one end of the ray (say, $I_{1}^{\text {inc }}$ ). The incident 
0 30
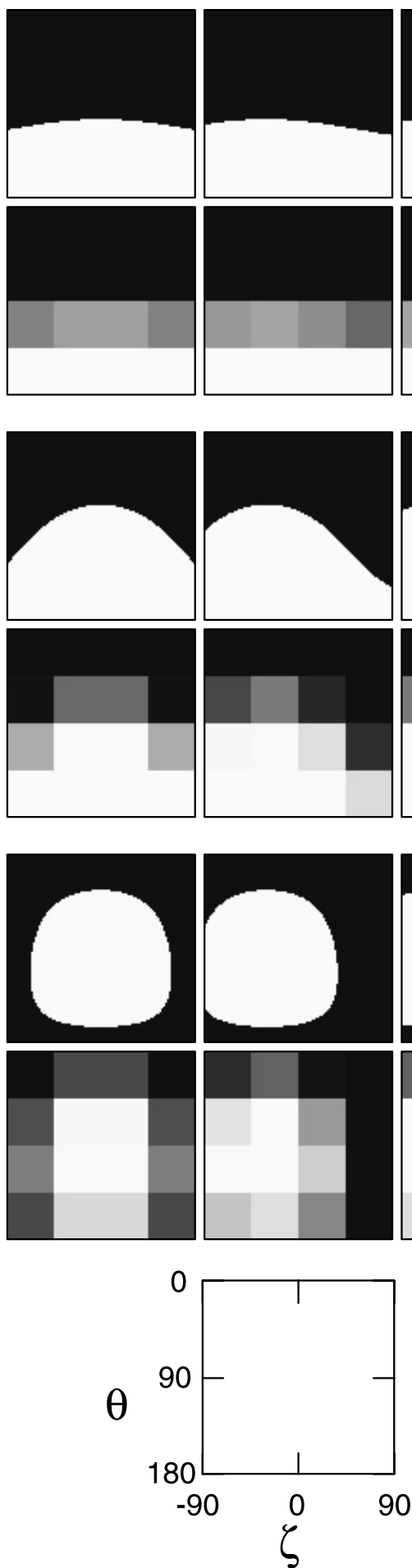

60
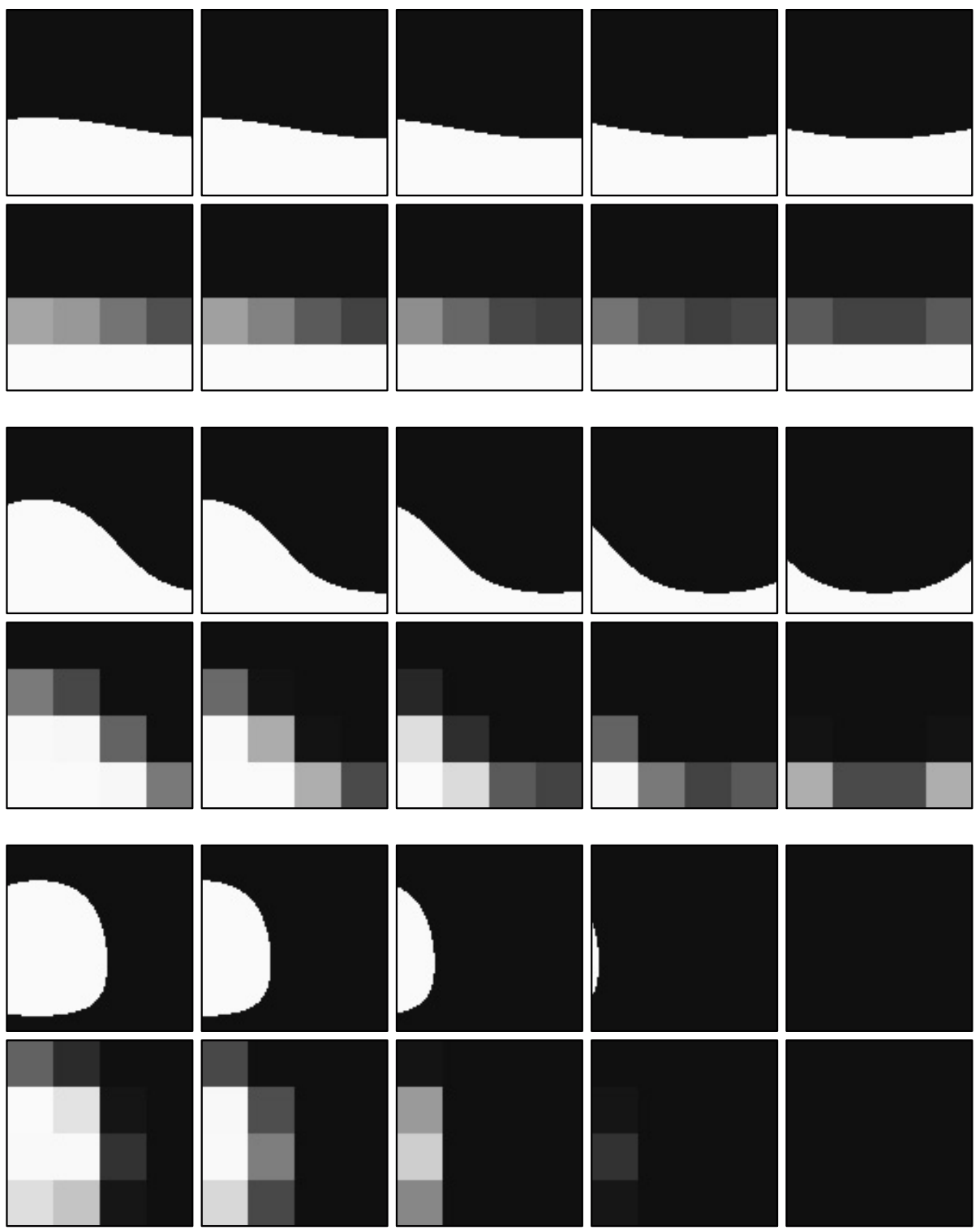

150

180
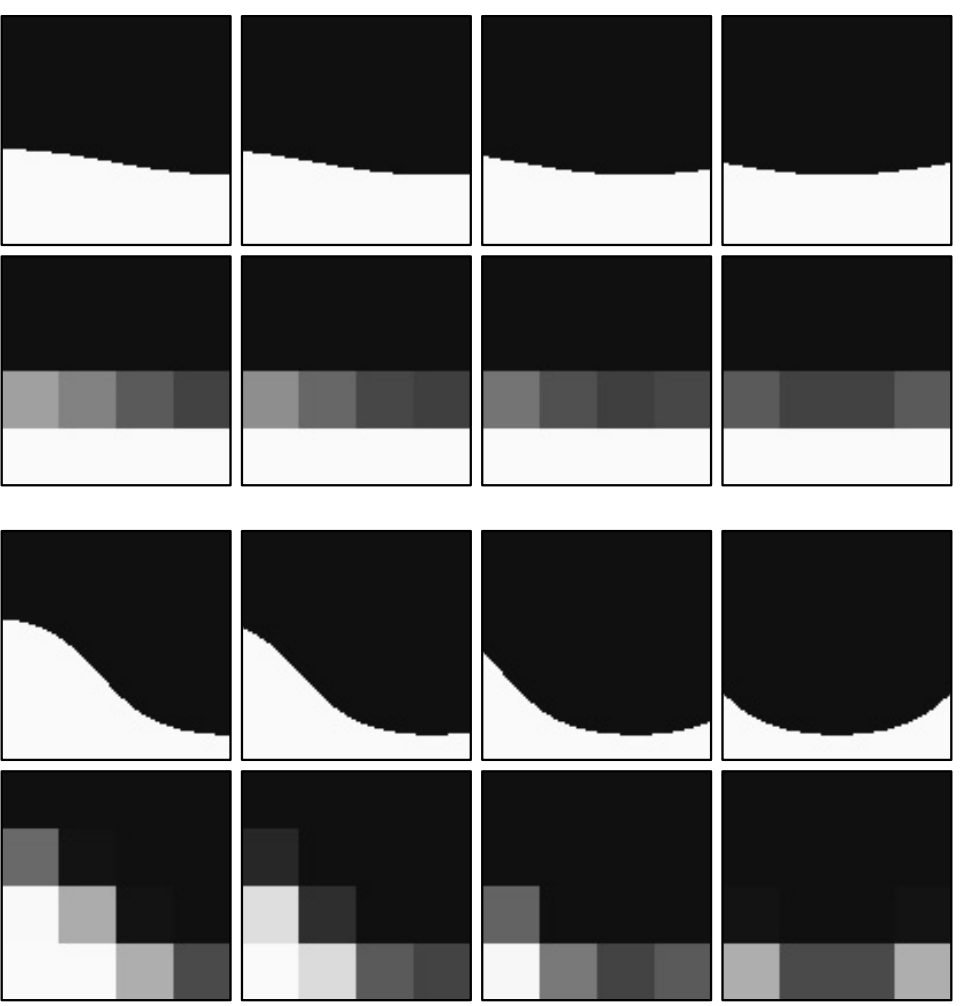

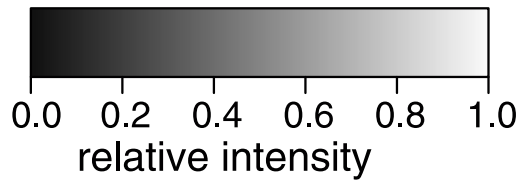

Fig. 3. Incident radiation received by a point of the cylinder, as a function of direction angles $\zeta$ and $\theta$. Each box corresponds to a given value of the inclination angle $\alpha$ and of the azimuthal angle $\psi$. From top to bottom: rows 1 and 2: $\alpha=10^{\circ}$ (nearly vertical); rows 3 and 4: $\alpha=45^{\circ}$; rows 5 and 6: $\alpha=80^{\circ}$ (nearly horizontal). Rows 1,3 , and 5 correspond to fine grids $(100 \times 100)$ in $\zeta$ and $\theta$, and rows 2,4 , and 6 to averaged coarse grids $(4 \times 4)$. From left to right, $\psi$ varies from $0^{\circ}$ to $180^{\circ}$ ( $\psi$ values are indicated at the top of the figure). The square at the bottom of the figure gives the scales of $\zeta$ and $\theta$ applicable to each box. The altitude is fixed to $70000 \mathrm{~km}$ and no limb-darkening is assumed.

intensity $I_{2}^{\text {inc }}$ in the opposite direction, necessary to solve numerically the radiative transfer equation, is obtained by using $\pi-\gamma$ instead of $\gamma$ in the preceding calculation.

\subsection{Azimuthal interpolation}

Since the problem under investigation is a 2D type transfer problem, two location variables $(r$ and $\psi$ ) need to be 
discretized. The $r$-mesh is similar to that used in the 1D case; i.e. the step is constant near the axis of the cylinder, and decreases exponentially in the external region (cf. Paper I). As will be seen later, we use intersections of the ( $r=$ const.) cylinders with the ray to discretize the transfer equation, so that no interpolation in $r$ is needed. The $\psi$-mesh has constant steps. Since the radiation field is symmetrical with respect to the plane $(\psi=0)$, this mesh is restricted to the range $[0, \pi]$, say,

$\psi_{j}=\pi \frac{j-1}{N_{\psi}-1}, \quad\left(j=1, \cdots, N_{\psi}\right)$.

To determine a quantity $F$ (absorption coefficient or source function) at any value of $\psi$ knowing its value at the points of the mesh $\left(F_{j}=F\left(\psi_{j}\right), j=1, \cdots, N_{\psi}\right)$, a procedure of interpolation by Fourier series is used:

$F(\psi)=\sum_{j=1}^{N_{\psi}} a_{j} \cos [(j-1) \psi]$

where the coefficients $a_{j}$ are computed from the $F_{j}$. Equation (8) yields a linear system for these coefficients

$\sum_{k=1}^{N_{\psi}} b_{j k} a_{k}=F_{j}, \quad\left(j=1, \cdots, N_{\psi}\right)$

with

$b_{j k}=\cos \left[(k-1) \psi_{j}\right]$.

After inversion of the matrix $\|b\|\left(\|B\|=\|b\|^{-1}\right)$, we have

$a_{j}=\sum_{k=1}^{N_{\psi}} B_{j k} F_{k}, \quad\left(j=1, \cdots, N_{\psi}\right)$.

The coefficients $b$ and $B$ only depend on the $\psi$ mesh and may be computed once and for all. This procedure of interpolation by Fourier series has the double advantage of smoothness and periodicity. Other smooth repesentations (e.g. splines) could also be adequate, while in contrast a simple linear interpolation procedure does not seem precise enough in the present case.

\subsection{Discretization of the ray equation}

In addition to $r$ and $\psi$, we introduce three other meshes for the direction variables $\zeta$ and $\theta$ and frequency $v$. The meshes for $\zeta$ and $\theta$ correspond to the coarse grid for incident radiation mentioned in Sect. 2.2. The mesh for $v$ is the same as used in Paper I.

Equation (2) is discretized in a straightforward way, using as mesh points the intersections of the ray with the cylinders corresponding to the radius mesh. The number of these intersections varies with each ray. If we consider a ray defined by the parameters $r_{j}, \psi_{k}, \zeta_{\alpha}$, and $\theta_{\beta}$, its shortest distance to the axis is:

$r_{\perp}(j, \alpha)=\left|r_{j} \sin \zeta_{\alpha}\right|$.

This defines an index "imin" such that

$r_{\text {imin-1 }} \leq r_{\perp}(j, \alpha)<r_{\text {imin }}$.
Since the values of $\kappa$ and $S$ are not symmetric as in the 1D cylindrical case, we use the full length of the ray, so that the number of intersection points is:

$\max =2 N-2$ imin +2.

For a given value of the index $s$ along the path $(1 \leq s \leq \max )$, the variable $h$ is obtained as:

$h_{s}= \begin{cases}-\sqrt{r_{N-s+1}^{2}-r_{\perp}^{2}}, & \text { if } \quad s \leq N-\mathrm{imin}+1 \\ \sqrt{r_{2 \mathrm{imin}-N-2+s}^{2}-r_{\perp}^{2}}, & \text { if } \quad s \geq N-\mathrm{imin}+2 .\end{cases}$

If we replace in Eq. (2) the derivatives by finite differences and rearrange the terms, we obtain a tridiagonal system of linear equations (we omit the index $v$ for simplicity).

For $2 \leq s \leq \max -1$

$-A_{s} Y_{s-1}+\left(1+A_{s}+C_{s}\right) Y_{s}-C_{s} Y_{s+1}=S_{s}$

with

$A_{s}=\frac{4 \sin ^{2} \theta}{\kappa_{s}\left(h_{s+1}-h_{s-1}\right)\left(\kappa_{s-1}+\kappa_{s}\right)\left(h_{s}-h_{s-1}\right)}$

and

$C_{s}=\frac{4 \sin ^{2} \theta}{\kappa_{s}\left(h_{s+1}-h_{s-1}\right)\left(\kappa_{s}+\kappa_{s+1}\right)\left(h_{s+1}-h_{s}\right)}$.

The first and last equations of the system are provided by boundary conditions (3). We obtain

$\left(1+C_{1}+H_{1}\right) Y_{1}-C_{1} Y_{2}=S_{1}+H_{1} I_{1}^{\text {inc }}$,

with $C_{1}=2 \sin ^{2} \theta /\left[\kappa_{1}^{2}\left(h_{2}-h_{1}\right)^{2}\right]$ and $H_{1}=2 \sin \theta /\left[\kappa_{1}\left(h_{2}-h_{1}\right)\right]$. At the other end of the ray, we similarly obtain

$-A_{\max } Y_{\max -1}+\left(1+A_{\max }+H_{\max }\right) Y_{\max }=S_{\max }+H_{\max } I_{2}^{\mathrm{inc}}$,

with $A_{\max }=2 \sin ^{2} \theta /\left[\kappa_{\max }^{2}\left(h_{\max }-h_{\max -1}\right)^{2}\right]$ and $H_{\max }=$ $2 \sin \theta /\left[\kappa_{\max }\left(h_{\max }-h_{\max -1}\right)\right]$.

Computation of coefficients in Eqs. (16) to (20) requires the determination of $\kappa$ and $S$ at each point of index $s$ along the ray. Radius $r$ may be recovered from Eq. (15), as $r_{N-s+1}$ in the first part of the ray, and $r_{2 \text { imin-N-2+s }}$ in the second part. The azimuth $\psi$ at the same point of the ray is obtained by a simple geometrical construction, with the result

$\psi(s)= \begin{cases}\psi_{k}+\zeta_{\alpha}-\frac{\pi}{2}-\arctan \left(\frac{h_{s}}{r_{\perp}}\right) & \text { if } \zeta_{\alpha}>0 \\ \psi_{k}+\zeta_{\alpha}+\frac{\pi}{2}+\arctan \left(\frac{h_{s}}{r_{\perp}}\right) & \text { if } \zeta_{\alpha}<0 .\end{cases}$

At this point, the interpolation procedure of Sect. 2.3 may be applied. Once the coefficients are computed, the tridiagonalsystem formed by Eqs. (16), (19), and (20) is solved for $Y$ 
using the procedure proposed by Rybicki \& Hummer (1991, Appendix A).

\subsection{Formal solution}

For a given point $(r, \psi)$ and a given frequency $v$, the $Y_{v}$ are computed as described in the preceding subsection for a set of directions $\left(\zeta_{\alpha}, \theta_{\beta}\right)$. Then, we obtain the mean intensity

$$
\begin{aligned}
J_{v}(r, \psi) & =\oint I_{v}(r, \psi, \zeta, \theta) \frac{\mathrm{d} \Omega}{4 \pi} \\
& =\frac{1}{2 \pi} \int_{-\pi / 2}^{\pi / 2} \mathrm{~d} \zeta \int_{0}^{\pi} Y_{\nu}(r, \psi, \zeta, \theta) \sin \theta \mathrm{d} \theta
\end{aligned}
$$

using the same quadrature formula as in Paper I, which gives

$J_{v}(r, \psi)=\sum_{\alpha=1}^{l} a_{\alpha} \sum_{\beta=1}^{m} b_{\beta} Y_{\nu}\left(r, \psi, \zeta_{\alpha}, \theta_{\beta}\right)$.

\section{Results for a two-level atom}

The transition between the two levels of the atom may be either a line or a bound-free continuum. As usual, we consider a line here, but the formulation for a continuum is very similar. In addition, we neglect continuous absorption and assume complete frequency redistribution. In this case, the source function is given by the usual expression

$S=\epsilon B+(1-\epsilon) \int_{0}^{\infty} J_{v} \phi_{v} \mathrm{~d} v$

where $\epsilon$ is the collisional quenching factor, $B$ the Planck function and $\phi_{v}$ the normalized Voigt profile $\left(\sqrt{\pi} \Delta v_{\mathrm{D}}\right)^{-1} H\left(a, \Delta v / \Delta v_{\mathrm{D}}\right)$ with damping factor $a$ and Doppler width $\Delta v_{\mathrm{D}}$. All these parameters may vary with $r$ and $\psi$; however, for simplicity, we use constant values in the following numerical applications: $\epsilon=10^{-4}, B=1$ and $a=10^{-3}$.

\subsection{Convergence properties}

Three methods are compared:

- pure $\Lambda$-iteration (PLI): we start from a given (arbitrary) value of the source function, compute the intensities along the different rays as shown in Sect. 2, integrate according to Eq. (22), and get a new source function with Eq. (24). Then the process is iterated.

- accelerated $\Lambda$-iteration (ALI): in addition to the preceding computations, it is necessary to obtain an approximation of the diagonal $\Lambda^{*}$ of the $\Lambda$-operator. This is done by using the coefficients $A$ and $C$ of Eq. (16) and applying to them the method proposed by Rybicki \& Hummer (1991, Appendix B). For each frequency in the line, we obtain contributions of different rays and integrate them with respect to $\zeta$ and $\theta$ to obtain $\Lambda_{v}^{*}$, which is the diagonal of the operator $\Lambda_{v}$ such that

$J_{v}=\Lambda_{v} S+\Gamma_{v}$
( $\Gamma_{v}$ is the contribution of the incident radiation, which is independent of $S$ : see Paper I). Then, instead of using Eq. (24), we iterate the source function as follows:

$$
S^{(m)}=\frac{\epsilon B+(1-\epsilon) \int_{0}^{\infty}\left(J_{v}-\Lambda_{v}^{*} S^{(m-1)}\right) \phi_{v} \mathrm{~d} v}{1-(1-\epsilon) \int_{0}^{\infty} \Lambda_{v}^{*} \phi_{v} \mathrm{~d} v},
$$

where $m$ indicates the iteration number.

- ALI + Ng acceleration: the process is the same as for ALI, but every four iterations, we replace the source function computed according to Eq. (26) by an "accelerated" source function. This one is a linear combination of the source functions involved in the three preceding iterations, as proposed by $\mathrm{Ng}$ (1974) and reported by Olson et al. (1986).

Results are shown in Fig. 4 for a cylinder whose axis makes an angle $\alpha=60^{\circ}$ with the vertical. Its optical thickness at line center, along a radius, is fixed at $10^{5}$, and its altitude is $H=70000 \mathrm{~km}$. The source functions are represented along a diameter but, in order to show their variations near the edges of the cylinder, a nonlinear scale is adopted; each interval of the $r$-mesh is represented by an equal length in the figure, while, in fact, these intervals are decreasing exponentially near the edges. The representation is realistic in the central part of the cylinder only, since the $r$-mesh is linear there.

In the left part of the figure, the results are shown for $I_{\mathrm{em}}(\mu)=0$. In this case, the problem degenerates into a 1D transfer problem, so we can compare the results of the $2 \mathrm{D}$ code with that of the $1 \mathrm{D}$ code and verify that the agreement is good. We represent the 25 first iterations starting either from $S=0$ or from an upper limit $(S=B=1)$. In the case of PLI (top of the figure), the convergence is far from being achieved, as shown by the large gap between upward and downward iterations. For accelerated $\Lambda$-iteration (operator splitting only), the convergence is practically achieved after 25 iterations, although small gaps remain near the edges. For $\mathrm{ALI}+\mathrm{Ng}$ acceleration, complete convergence is achieved after a dozen iterations.

In the right part of the figure, we represent the corresponding iterations for a solar emission $I_{\mathrm{em}}(\mu)=10$, which is significantly higher than the Planck function inside the cylinder. As a result of the large inclination angle $\left(\alpha=60^{\circ}\right)$, the different parts of the cylinder receive very different incident radiations, with a maximum for $\psi=0^{\circ}$ and a minimum for $\psi=180^{\circ}$. Thus, the final source function is decreasing along the diameter, which is chosen in such a way that its first half corresponds to $\psi=0^{\circ}$ and the second one to $\psi=180^{\circ}$. As far as convergence is concerned, conclusions are the same as in the preceding case. For downward iterations, the starting value was taken as $S=8$, in order to have an uniform decrease of source functions.

\subsection{Effect of the inclination angle}

The big difference between the present code and those described in Paper I is its ability to model flux tubes above the solar surface, the axes of which are not vertical. This improvement is offset by the longer computation time, caused mainly by the need to use a $2 \mathrm{D}$ grid ( $r$ and $\psi$ ), making important to 

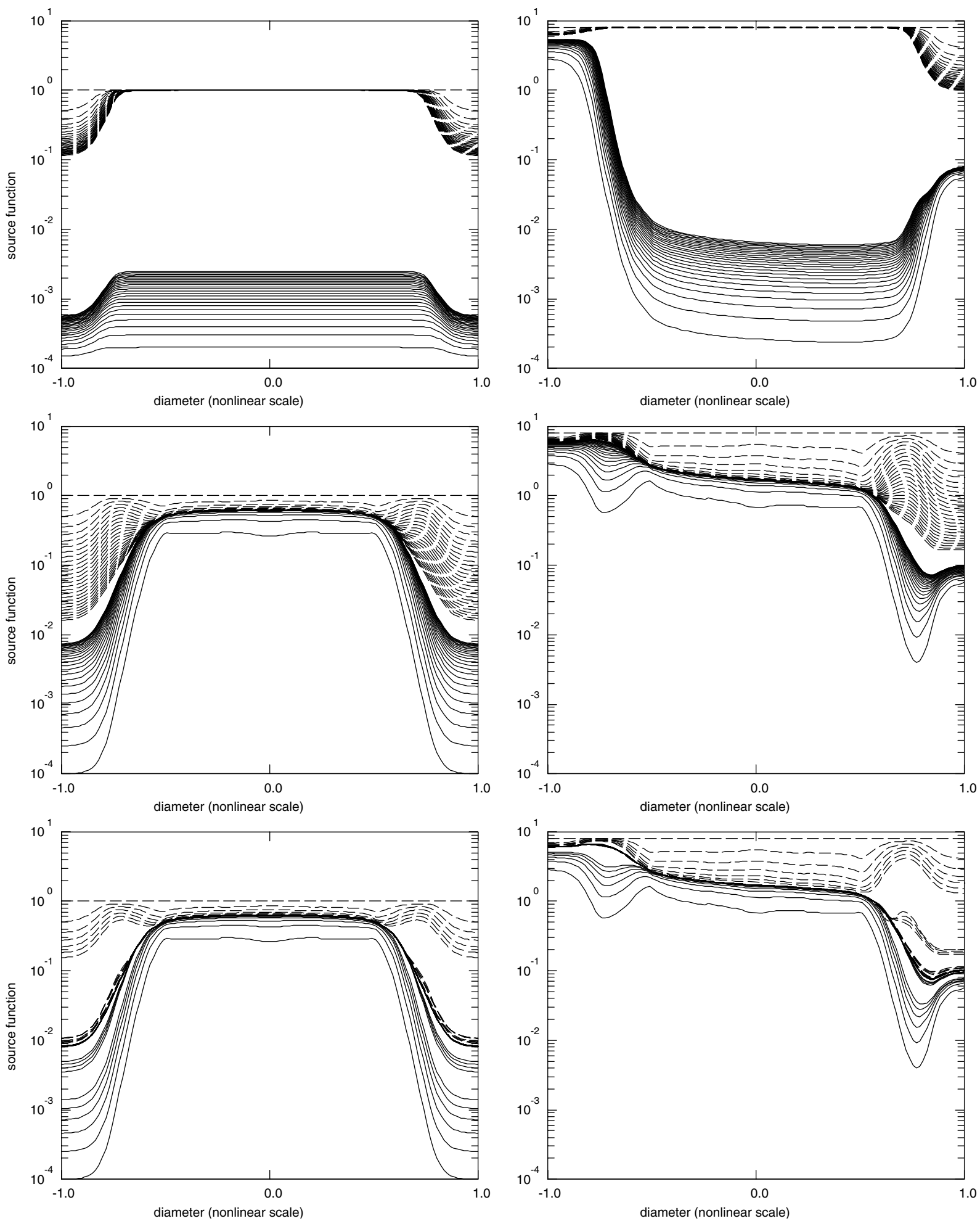

Fig. 4. Source function evolution during iterations. Left column: no incident radiation. Right column: incident radiation characterized by $I_{\mathrm{em}}(\mu)=$ $10, H=70000 \mathrm{~km}$ and $\alpha=60^{\circ}$. Upper row: pure $\Lambda$-iteration. Middle row: ALI (operator splitting only). Lower row: ALI $+\mathrm{Ng}$ acceleration. Abscissae represent the location along a diameter, with a nonlinear scale, as described in the text. Upward iterations (starting from $S=0$ ) are represented by solid lines. Downward iterations (starting from either $S=1$ or $S=8$ ) are represented by dashed lines.

examine the influence of the inclination angle $\alpha$ on the results, at least to evaluate the errors produced by the $1 \mathrm{D}$ approximation. To this purpose, we keep the model used in the preceding section, with $\kappa=1$ at line center, a radius $R=10^{5}$, and parameters $\epsilon=10^{-4}, B=1$, and $a=10^{-3}$. Incident intensity is also defined by $I_{\mathrm{em}}(\mu)=10$, and altitude $H=70000 \mathrm{~km}$. Angle $\alpha$ varies from nearly vertical to nearly horizontal values. Figure 5 shows the source functions vs. $r$ and $\psi$, for $\alpha=5^{\circ}, 25^{\circ}$, 

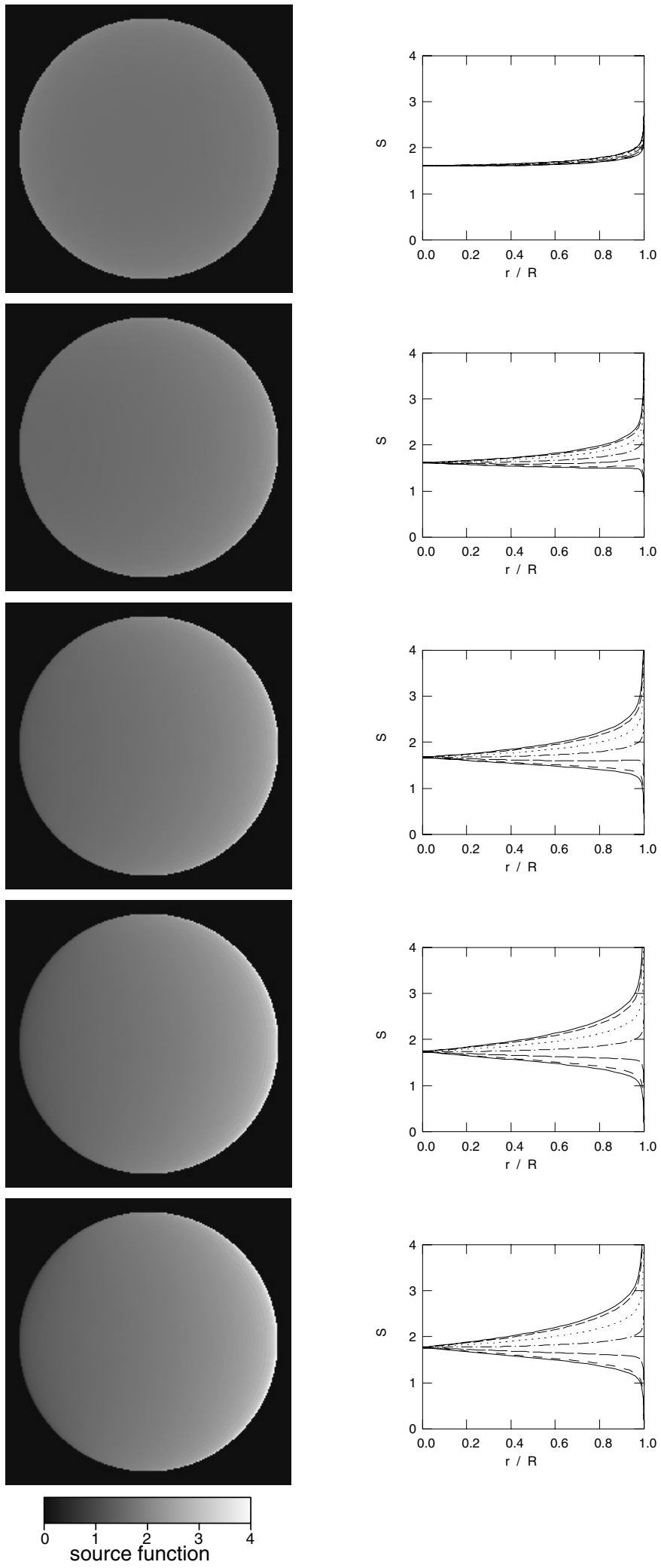

Fig. 5. Variations of the source function inside a normal section of the cylinder. The model is the same as in the right part of Fig. $4\left(I_{\mathrm{em}}(\mu)=\right.$ $10, H=70000 \mathrm{~km})$, but the inclination angle varies: from top to bottom: $\alpha=5^{\circ}, 25^{\circ}, 45^{\circ}, 65^{\circ}$ and $85^{\circ}$. The same source functions are plotted vs. $r$ in the right part of the figure, for 7 values of $\psi: 0^{\circ}, 30^{\circ}$, $60^{\circ}, 90^{\circ}, 120^{\circ}, 150^{\circ}$, and $180^{\circ}$. The order of curves is not indicated but, in every case, $\mathrm{S}$ decreases as $\psi$ increases. $45^{\circ}, 65^{\circ}$, and $85^{\circ}$. These results are presented in two forms: as two-dimension images on the left, and as plots of $S(r)$ on the right, each curve corresponding to a given value of $\psi$. In this example, it appears that the source function along the axis is practically independent of $\alpha$, while the source function near the edge shows different behaviours according to the value of $\alpha$. As expected, $S$ is practically independent of $\psi$ for $\alpha=5^{\circ}$, but varies by a factor of about 3 in the case $\alpha=85^{\circ}$. Finally, it appears that the $2 \mathrm{D}$ code is necessary for the precise modelling of inclined tubes, while the $1 \mathrm{D}$ code may provide a rough approximation, or may be used to obtain an initial value for iterations.

Timing: Computation time depends on several parameters, especially the number of iterations $N_{\text {it }}$, the sizes of spatial grids $N_{r}$ and $N_{\psi}$, direction grids $N_{\theta}$ and $N_{\zeta}$, and frequency grid $N_{v}$. The time measured for $N_{\text {it }}=25, N_{r}=61, N_{\psi}=7, N_{\theta}=4$, $N_{\zeta}=4$, and $N_{v}=25$ was 773 s on a PC of year 2001 with a clock frequency of $697 \mathrm{MHz}$. As the main part of the time is spent solving the radiative transfer equation along the rays, this time should be approximately proportional to $N_{\text {it }}, N_{\psi}, N_{\theta}$, $N_{\zeta}$, and $N_{v}$. An increase in the parameter $N_{r}$ produces at the same time an increase in the number of rays and of intersections along each ray, so that the computing time should scale approximately as $N_{r}^{2}$.

\section{Conclusion}

The program described in this paper constitutes a step towards constructing more complex codes to allow realistic modelling of complex loop systems, as observed in the solar corona (especially in $\mathrm{H} \alpha$ and in EUV lines). With its 2D structure, it is able to deal with tubes whose axes are inclined in an arbitrary way, so we could use it to treat successive segments of a loop. For the moment this program is restricted to a two-level atom and complete frequency redistribution. The next step will be an extension to multilevel atom, with application to hydrogen and correction of electron density, as was done for the 1D code in Paper I. Further investigations should include the helium atom, plus some elements necessary to modelling existing observations. We also plan to implement partial frequency redistribution for the treatment of several resonance lines.

\section{References}

Gouttebroze, P. 1990, A\&A, 228, 295 (G90)

Gouttebroze, P. 2004, A\&A, 413, 733 (Paper I)

Ng, K. C. 1974, J. Chem. Phys., 61, 2680

Olson, G. L., Auer, L. H., \& Buchler, J. R. 1986, JQSRT, 35, 431

Rybicki, G. B., \& Hummer, D. G. 1991, A\&A, 245, 171 